Note: This is a preprint of a paper being submitted for publication. Contents of this paper should not be quoted nor referred to without permission of the author(s).

\title{
Spectroscopic Ellipsometry Characterization of Thin-Film Silicon Nitride
}

G. E. Jellison, Jr., and F. A. Modine

Solid State Division Oak Ridge National Laboratory Oak Ridge, TN 37831-6056

P. Doshi,* and A. Rohatgi*

*Georgia Institute of Technology Atlanta, GA 30332-0250

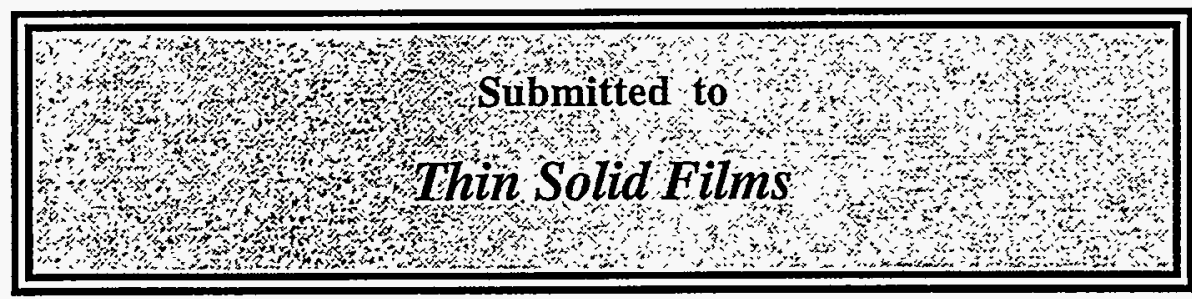

The submitted manuscript has been authored by a contractor of the U.S. Government under contact DE-ACOS-960R22464. Accordingly, the U.S. Government retains a nonexclusive, the U.S. Government retains a nonexclusive, published form of this contribution, or allow others to do so, for U.S. Government purposes."

\section{DISTRIBUTION OF THIS DOCUMENT ISUNLIMH H}

May 1997

Prepared by

Solid State Division

Oak Ridge National Laboratory

P.O. Box 2008

Oak Ridge, Tennessee 37831-6056

managed by

LOCKHEED MARTIN ENERGY RESEARCH CORP.

for the

U.S. DEPARTMENT OF ENERGY

under contract DE-AC05-96OR22464 


\section{DISCLAIMER}

This report was prepared as an account of work sponsored by an agency of the United States Government. Neither the United States Government nor any agency thereof, nor any of their employees, make any warranty, express or implied, or assumes any legal liability or responsibility for the accuracy, completeness, or usefulness of any information, apparatus, product, or process disclosed, or represents that its use would not infringe privately owned rights. Reference herein to any specific commercial product, process, or service by trade name, trademark, manufacturer, or otherwise does not necessarily constitute or imply its endorsement, recommendation, or favoring by the United States Government or any agency thereof. The views and opinions of authors expressed herein do not necessarily state or reflect those of the United States Government or any agency thereof. 


\section{DISCLAMIER}

Portions of this document may be illegible in electronic image products. Images are produced from the best available original document. 


\title{
Spectroscopic ellipsometry characterization of thin-film silicon nitride
}

\author{
G. E. Jellison, Jr. and F. A. Modine \\ Solid State Division, Oak Ridge National Laboratory, \\ Oak Ridge, TN 37831-6030 \\ and \\ P. Doshi and A. Rohatgi \\ School of Electrical and Computer Engineering \\ Georgia Institute of Technology, Atlanta, GA 30332-0250
}

\begin{abstract}
We have measured and analyzed the optical characteristics of a series of silicon nitride thin films prepared by plasma-enhanced chemical vapor deposition on silicon substrates for photovoltaic applications. Spectroscopic ellipsometry measurements were made by using a two-channel spectroscopic polarization modulator ellipsometer that measures $N, S$, and $C$ data simultaneously. The data were fit to a model consisting of air / roughness / SiN / crystalline silicon. The roughness was modeled using the Bruggeman effective medium approximation, assuming $50 \% \mathrm{SiN}, 50 \%$ voids. The optical functions of the SiN film were parameterized using a model by Jellison and Modine [Appl. Phys. Lett. 69, 371 (1996); 69, 2137 (1996).]. All the $\chi^{2}$ are near 1, demonstrating that this model works extremely well for all SiN films. The measured dielectric functions were used to make optimized $\mathrm{SiN}$ antireflection coatings for crystalline silicon solar cells.
\end{abstract}




\section{Introduction}

Amorphous silicon nitride has found a large number of uses in the semiconductor industry. However, it has been recognized for some time that this material may have greater impact if the quality of $\mathrm{SiN}$ films can be improved to the point where they could be employed as gate dielectrics. ${ }^{1.2}$ This is because $\mathrm{SiN}$ has a number of characteristics that make it more attractive than the $\mathrm{SiO}_{2}$ films, which are presently used: specifically, $\mathrm{SiN}$ has a higher dielectric constant than $\mathrm{SiO}_{2}$ (7.5 versus 3.9$)$, and it is generally more resistant to impurity diffusion. SiN is not yet used for gate dielectrics because conventionally grown $\mathrm{SiN}$ has a high density of electronic defects, both in the bulk and at the $\mathrm{Si} / \mathrm{N}$ interface.

The refractive index of SiN is greatly dependent upon deposition conditions, but is greater than 2.0 at $630 \mathrm{~nm}$, which makes it an ideal candidate for single-layer antireflection coatings on amorphous or crystalline silicon solar cells. ${ }^{3.45}$ The SiN films are impervious to moisture, unlike common alternatives such as $\mathrm{ZnS} / \mathrm{MgF}_{2}$ films. Furthermore, it has been shown that annealing hydrogenated SiN films releases some hydrogen, ${ }^{4}$ which then may passivate some of the defects at the interface and in the bulk, further improving the solar cell performance.

Since the optical and other physical properties of thin-film SiN vary considerably with deposition conditions, there is a need for a simple, non-destructive diagnostic technique that is sensitive to the quality of the SiN film. In this paper, we discuss the use of spectroscopic ellipsometry (SE) as such a diagnostic for SiN films. The large variation of the optical properties of SiN films makes systematic interpretation of the SE data 
difficult without a descriptive model. Recently, a model has been developed ${ }^{6}$ that provides a general parametric description of the optical functions of amorphous materials using only 4 or 5 parameters. Here, we apply this model to SE data taken on a series of SiN films grown using plasma-enhanced chemical vapor deposition. Using the parameters from this model, we have been able to characterize the growth system and predictably grow films with particular refractive index characteristics. These data have also been used to determine the optimum film thickness for photovoltaic applications. ${ }^{5}$

\section{Experiment}

SiN films were deposited on polished silicon samples in the Plasma-Therm PECVD system operating at $13.6 \mathrm{MHz}$. Deposition conditions included a pressure of 0.9 torr, power of $20 \mathrm{~W}$, and temperature of $300^{\circ} \mathrm{C}$. The refractive index of SiN was varied by controlling the $\mathrm{SiH}_{4}$ to $\mathrm{NH}_{3}$ flow rate ratio. Several films were also annealed at temperatures between 550 and $750 \mathrm{C}$ from 10 to $180 \mathrm{sec}$ using rapid thermal annealing. The main application for these films is as anti-reflection coatings for silicon solar cells, so the film thicknesses varied from 50 to $80 \mathrm{~nm}$.

SE measurements were made using a 2-channel spectroscopic polarization modulation ellipsometer ${ }^{7}$ from 250 to $840 \mathrm{~nm}$. This instrument measures the associated ellipsometric parameters for isotropic samples 


$$
\begin{aligned}
& N=\cos (2 \psi) \\
& S=\sin (2 \psi) \sin (\Delta) \\
& C=\sin (2 \psi) \cos (\Delta)
\end{aligned}
$$

where the angles $\psi$ and $\Delta$ are the standard ellipsometric angles representing the change in amplitude and phase shift, respectively, upon reflection. If the films are not depolarizing, then $N^{2}+S^{2}+C^{2}=1$ and it is appropriate to convert the SE data to the $\rho$ representation, which is given by

$$
\rho=\frac{r_{p}}{r_{s}}=\tan \psi e^{i \Delta}=\frac{C+i S}{1+N}
$$

where $r_{p}\left(r_{s}\right)$ is the complex reflection coefficient for light polarized parallel (perpendicular) to the plane of incidence. A sample $\rho$ spectrum is shown in Fig. 1.

\section{Analysis of Spectroscopic ellipsometry data}

- As mentioned in the introduction, one of the primary needs for SE measurements is for the availability of realistic models for the dielectric functions of materials. The literature has many such examples, but one that has received considerable attention during the last few years is a model proposed by Forouhi and Bloomer $(F \& B)^{8}$. This model starts with a derived expression for the extinction coefficient, given by 


$$
k_{F B}(E)=\frac{A\left(E-E_{g}\right)^{2}}{E^{2}-B E+C}
$$

where $A, B, C$ and $E_{g}$ are treated as fitting parameters. The refractive index was obtained from $k_{F B}(E)$ using Kramers-Kronig integration, where a term $n(\infty)$ was included as an additional fitting parameter.

Although the F\&B formulation appears to fit several $n$ and $k$ data sets using the "chi-by-eye" criteria, there are several fundamental problems:

1) $k_{F B}(E)>0$ for $E<E_{g}$. Clearly, interband transitions cannot result in optical absorption for $E<E_{g}$; after all, many glasses are transparent in the visible.

2) As $E \rightarrow \infty$ then $k_{F B}(E) \rightarrow{\text { constant. Both experimental }{ }^{9} \text { and theoretical }}^{10}$ results clearly indicate that $k(E) \rightarrow 0$ as $1 / E^{3}$ or faster as $E \rightarrow \infty$.

3) $\mathrm{F} \& \mathrm{~B}$ did not use time-reversal symmetry in their calculation of $n_{F B}(E)$ from the Kramers-Kronig integration of $k_{F B}(E)$; this requires that $k(-E)=-$ $k(E)$.

Moreover, detailed fitting of several data sets found in the literature showed that the F\&B formalism did not fit the published data. ${ }^{6}$

A more realistic model ${ }^{6}$ of the optical functions of amorphous materials is based on the Tauc joint density of states and the Lorentz model for the dielectric response for a collection of single atoms. If only a single transition is considered, then the imaginary part of the dielectric function is given by 


$$
\begin{aligned}
\varepsilon_{2 T L} & =\frac{A E_{o} C\left(E-E_{g}\right)^{2}}{\left(E^{2}-E_{o}^{2}\right)^{2}+C^{2} E^{2}} \frac{1}{E} & & E>E_{g} \\
& =0 & & E \leq E_{g}
\end{aligned}
$$

where $E_{o}$ is the peak transition energy, $C$ is the broadening term, $E_{g}$ is the optical band edge, and $A$ is proportional to the transition probability matrix element. The real part of the dielectric function is given by the Kramers-Kronig integral of Eq. 4:

$$
\varepsilon_{1 T L}(E)=\varepsilon_{1}(\infty)+\frac{2}{\pi} P \int_{E_{g}}^{\infty} \frac{\xi \varepsilon_{2 T L}(\xi)}{\xi^{2}-E^{2}} d \xi
$$

where $\varepsilon_{l}(\infty)$ has been added as an integration constant. In general, it is expected that $\varepsilon_{l}(\infty)=1$. In contrast to the F\&B formalism, the Tauc-Lorentz expressions satisfy the criteria 1) to 3) above, and they do fit the published data much better. ${ }^{6}$

The model used to fit the spectroscopic ellipsometry data taken on all SiN films was air / surface roughness / SiN / c-Si. The surface roughness was modeled using a Bruggeman effective medium approximation ${ }^{11}$ consisting of $50 \%$ voids and $50 \% \mathrm{SiN}$. The optical functions of SiN were parameterized using the Tauc-Lorentz model described above and in ref. 6 , and the optical functions of crystalline silicon were taken from ref. 12. The fitting was performed using a Levenberg-Marquardt algorithm, where 6 parameters were fitted: the roughness thickness, the film thickness, and 4 parameters from the Tauc-Lorentz model $\left(\varepsilon_{l}(\infty)\right.$ is set to 1$)$. The reduced $\chi^{2}$ was used as a figure of merit, and all errors and cross-correlation coefficients were calculated based on the actual 
experimental errors. ${ }^{13}$ A sample fit to the data is shown in Fig. 1, and the details of the fitted parameters obtained from the fit are shown in Table I, including the correlated and uncorrelated errors of the fitted parameters.

\section{Discussion}

Clearly, the fit shown in Figure 1 is a good fit. The reduced $\chi^{2}$ is less than 1 , and the correlated and uncorrelated errors (shown in Table I) are not large, meaning that there is very little correlation between the parameters and that all parameters can be separately determined. Moreover, the spectroscopic difference between the fitted $\rho$ and the calculated $\rho$ (shown in the two bottom panels of Fig. 1) shows that there is no spectral region where the fit is bad.

Table II shows a summary of the fitted parameters determined from 17 different SiN films grown using PE-CVD, and Figure 2 shows the refractive index (n) and extinction coefficient $(\mathrm{k})$ obtained from the fitted parameters for 5 representative amorphous SiN films. Clearly, this model fits a wide range of different amorphous SiN films (as evidenced by the wide variation in optical properties), and yields reasonable $\chi^{2}$ 's in all cases. Moreover, this is done with only four parameters to describe the optical functions of $\operatorname{SiN}$; the $\varepsilon_{l}(\infty)$ can be used as a fitting parameter (as was done in ref. 8), but this is unnecessary. Setting $\varepsilon_{/}(\infty)=1$ is clearly more physical and eliminates one of the more unsettling points that arose from the F\&B formulation, where they would routinely get values of $n(\infty)$ up to 2.7 ( or $\varepsilon_{l}(\infty)=7.3$ ). 
The six parameters that are determined from this fitting procedure could all be important in determining the quality of the film. The rough thickness and the film thickness are obviously important. However, the four parameters that are obtained from the Tauc-Lorentz model (particularly the band edge $E_{g}$ ) also have physical significance and are used to calculate the spectroscopic optical functions of the amorphous SiN films (see Fig. 2).

As can be seen from Table I, there is a strong anti-correlation between $n(630 \mathrm{~nm})$ and $E_{\Omega}$. There also is a correlation between $n(630 \mathrm{~nm})$ and $A$, and an anti-correlation between $n(630 \mathrm{~nm})$ and $E_{l}$. For this reason, we have chosen to use the value of $n(630$ $\mathrm{nm}$ ) as an alternate name for each of the films.

The total collected current from a solar cell is given by

$$
J=\int T(\lambda) N(\lambda) Q E(\lambda) d \lambda
$$

where $T(\lambda)$ is the wavelength-dependent transmission coefficient (the fraction of incident light that actually enters the cell), $N(\lambda)$ is the number of photons per $\mathrm{nm}$ per $\mathrm{cm}^{2}$ incident upon the cell (such as AM 1.5 Global), and $\mathrm{QE}(\lambda)$ is the internal quantum efficiency of the solar cell. If the cell is covered with an anti-reflection coating, then $T(\lambda)$ will increase toward unity, but will be spectrally dependent because of the wavelength-dependent optical functions and interference effects of the anti-reflection. Utilizing the optical functions for SiN obtained from these SE measurements, we were able to design an optimum thin film thickness for use as an anti-reflection coating ${ }^{5}$. For a single layer $\mathrm{SiN}$ 
AR coating in air, sample $3(n(630)=2.02)$ at a thickness of $78 \mathrm{~nm}$ gave the maximum amount of AM 1.5 light transmitted to the solar cell. If a double-layer AR coating of $\mathrm{SiN} / \mathrm{MgF}_{2}$ was used, sample $8((n(630)=2.23))$ at $64 \mathrm{~nm}$ proved to be the optimum material and thickness to give maximum transmission to the solar cell.

This research is sponsored by the Division of Materials Science, Oak Ridge National Laboratory, managed by Lockheed Martin Energy Research, for the U. S. Department of Energy, under Contract No. DE-AC05-960R22464 and by Sandia National Laboratories under Contract No. AO-6162. 


\section{References}

1. S. M. Sze, VLSI Technology, (McGraw-Hill, New York, 1983), p. 119.

2. Z. Lu, S. S. He, Y. Ma, and G. Lucovsky, J. Non-Cryst. Solids, 187 (1995) 340.

3. R. Kishore, S. N. Singh, and B. K. Das, Solar En. Mater. Solar Cells 26 (1992)

27.

4. L. Cai, D. Yang, M. A. El-Sayed, and A. Rohatgi, J. Appl. Phys., 80 (1996) 5384.

5. P. Doshi, A. Rohatgi, and G. E. Jellison, Jr., submitted to Appl. Opt. (1997).

6. G. E. Jellison, Jr. and F. A. Modine, Appl. Phys. Lett., 69 (1996) 371; ibid. 69 (1996) 2137.

7. G. E. Jellison, Jr. and F. A. Modine, Appl. Opt., 29 (1990) 959.

8. A. R. Forouhi and I. Bloomer, Phys. Rev. B, 34 (1986) 7018.

9. Handbook of Optical Constants of Solids I edited by E. D. Palik (Academic, New York, 1985); Handbook of Optical Constants of Solids II edited by E. D. Palik (Academic, New York, 1991).

10. F. Wooton, Optical Properties of Solids (Academic, New York, 1972).

11. D. A. G. Bruggeman, Ann. Phys. (Leipzig), 24 (1935) 636.

12. G. E. Jellison, Jr., Opt. Materials, 1 (1992) 41.

13. G. E. Jellison, Jr., Appl. Opt., 30 (1991) 3354; G. E. Jellison, Jr., Thin Solid Films, 234 (1993) 416. 


\section{Table I}

Details of the fitted parameters obtained from the fit shown in Figure 1. The model consisted of 4 layers: air (0) / surface roughness (1) / SiN (2), modeled using the TaucLorentz model / crystalline silicon (3), data taken from ref. 12. The $\chi^{2}=0.23$.

\begin{tabular}{ccccc} 
Layer No. & Parameter & Value & \multicolumn{2}{c}{ Errors } \\
& & & uncorrelated & correlated \\
1 & Thickness (nm) & 2.01 & 0.06 & 0.32 \\
2 & Thickness (nm) & 28.79 & 0.03 & 0.17 \\
2 & $E_{g}(\mathrm{eV})$ & 2.527 & 0.0018 & 0.021 \\
2 & $A(\mathrm{eV})$ & 100.19 & 0.093 & 3.7 \\
2 & $B_{0}(\mathrm{eV})$ & 7.897 & 0.014 & 0.12 \\
& $C(\mathrm{eV})$ & 10.142 & 0.019 & 0.69
\end{tabular}




\section{Table II}

The resulting fitting parameters for a series of $17 \mathrm{SiN}$ samples examined in this study. Note that the larger values of $B_{o}$ and $C$ are listed to one less significant figure than the smaller values, indicating the lower accuracy of these values. The error limits are an average of the errors, where the error limits for the $B_{o}$ and $C$ terms include only those reported to 3 significant figures.

\begin{tabular}{|c|c|c|c|c|c|c|c|c|}
\hline Sample & $\begin{array}{c}\text { Refractive } \\
\text { Index } \\
(630 \mathrm{~nm})\end{array}$ & $\begin{array}{l}\text { Rough } \\
\text { Thickness } \\
\text { (nm) }\end{array}$ & $\begin{array}{l}\text { Film } \\
\text { Thickness } \\
\text { (nm) }\end{array}$ & $\begin{array}{c}E_{g} \\
(\mathrm{eV})\end{array}$ & $\begin{array}{c}A \\
(\mathrm{eV})\end{array}$ & $\begin{array}{c}B_{o} \\
(\mathrm{eV})\end{array}$ & $\begin{array}{c}C \\
(\mathrm{eV})\end{array}$ & $\chi^{2}$ \\
\hline L5 & 3.495 & 4.1 & 72.5 & 1.72 & 175 & 3.76 & 4.10 & 1.01 \\
\hline $\mathrm{L} A$ & 3.382 & 3.5 & 75.7 & 1.84 & 202 & 3.50 & 4.02 & 0.58 \\
\hline 1 & 2.972 & 4.4 & 60.7 & 1.94 & 164 & 4.24 & 5.20 & 0.89 \\
\hline L3 & 3.090 & 3.6 & 80.0 & 2.00 & 221 & 3.39 & 4.31 & 1.63 \\
\hline 2 & 2.892 & 4.2 & 46.3 & 2.02 & 177 & 4.30 & 5.9 & 0.56 \\
\hline 4 & 2.800 & 4.2 & 59.4 & 2.06 & 167 & 4.38 & 5.81 & 1.34 \\
\hline$E$ & 2.555 & 3.3 & 66.5 & 2.15 & 147 & 4.89 & 7.1 & 0.68 \\
\hline 7 & 2.361 & 3.7 & 51.5 & 2.18 & 122 & 6.58 & 10.5 & 1.04 \\
\hline 9 & 2.396 & 3.6 & 54.7 & 2.19 & 132 & 6.40 & 10.9 & 1.23 \\
\hline $\mathrm{D}$ & 2.432 & 2.9 & 73.6 & 2.21 & 136 & 5.36 & 8.15 & 0.58 \\
\hline 6 & 2.279 & 3.4 & 59.6 & 2.32 & 121 & 6.73 & 10.6 & 0.82 \\
\hline 8 & 2.230 & 3.2 & 56.8 & 2.33 & 107 & 6.9 & 9.5 & 0.72 \\
\hline 5 & 2.249 & 2.8 & 56.3 & 2.34 & 115 & 6.81 & 10.1 & 0.50 \\
\hline $\mathrm{C}$ & 2.205 & 2.0 & 60.6 & 2.41 & 113 & 6.73 & 9.9 & 0.22 \\
\hline B & 2.164 & 2.1 & 51.8 & 2.51 & 105 & 7.4 & 9.5 & 0.28 \\
\hline A & 2.120 & 2.0 & 58.8 & 2.53 & 100 & 7.02 & 9.7 & 0.89 \\
\hline 3 & 2.022 & 2.9 & 48.9 & 2.74 & 80 & 8.8 & 7.0 & 0.19 \\
\hline$<$ Error $>$ & ---- & 0.2 & 0.1 & 0.01 & 3 & 0.05 & 0.09 & \\
\hline
\end{tabular}




\section{Figure Captions}

1. The complex $\rho$ spectrum for sample A, showing the ellipsometric data (dots and triangles) and the fit to the data (line). The bottom two panels show the difference between the ellipsometric data and the fits, where the dots indicate the error limits of the data. The $\chi^{2}$ of the fit was 0.23 .

2. The refractive index $(n)$ and the extinction coefficient $(k)$ for 5 different SiN films. 

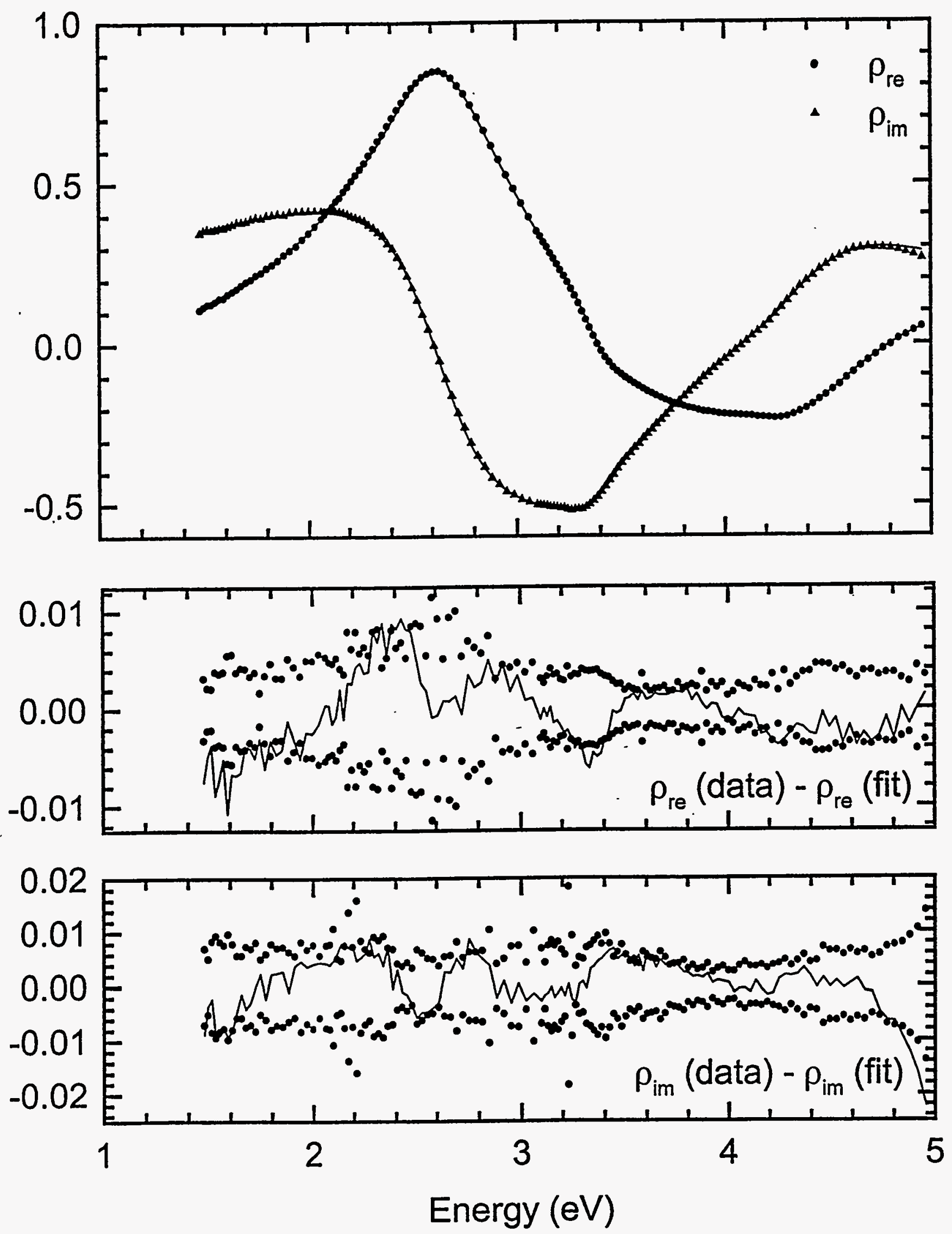

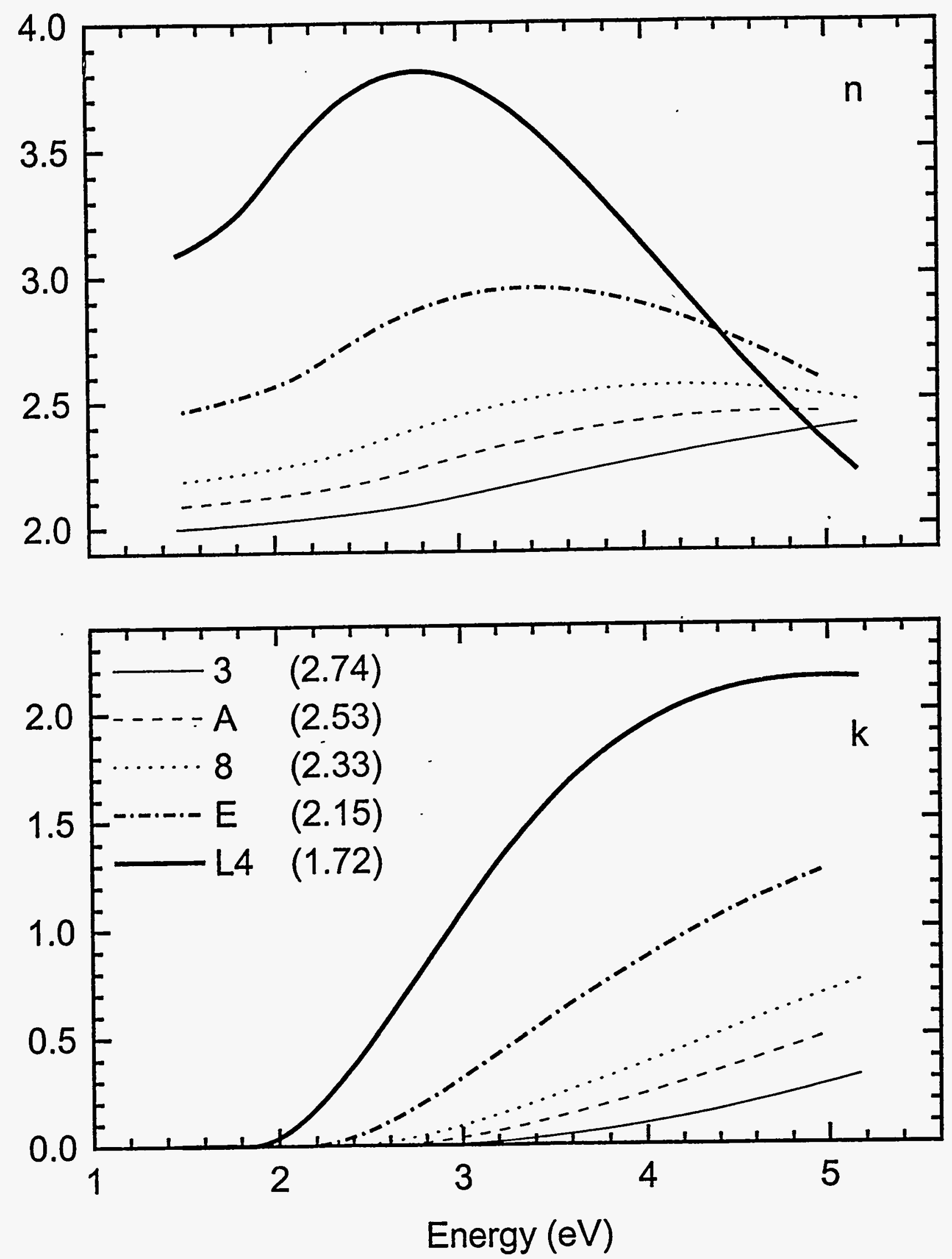